\title{
¿Sabemos qué es un crédito ECTS?
}

\author{
Josep Domenech y Desamparados Blazquez \\ Departamento de Economía y Ciencias Sociales, Universitat Politècnica de València \\ jdomenech@upvnet.upv.es; mdeblzso@upvnet.upv.es
}

\begin{abstract}
In the context of the European Higher Education Area (EHEA), universities base the design and evaluation of their study programs in the ECTS credits system. This system facilitates the comparability of university studies and, therefore, boosts students mobility. ECTS relies on credits as unit for measuring students' workload. This paper challenges this definition by analyzing the time students spent on a course at the Universitat Politècnica de València. These students have followed a continuous assessment method with weekly tests in which they were asked about how many hours they employed to prepare them. Results show that the real individual workload is much lower than the expected. Possible reasons and options to improve are discussed.
\end{abstract}

Keywords: ECTS, EEES, student individual workload

\section{Resumen}

En el contexto del Espacio Europeo de Educación Superior (EEES), las universidades basan el diseño y evaluación de sus planes de estudio en el sistema de créditos ECTS. Este sistema facilita la comparación de las titulaciones y así, la movilidad de los estudiantes. Sin embargo, se vienen observando deficiencias en su papel como elemento cuantificador de la carga de trabajo de los estudiantes. Así, en este artículo se expone un caso de estudio llevado a cabo en la Universitat Politècnica de València en el que se analiza la carga de trabajo esperada según la definición del crédito ECTS y la carga real reportada por los alumnos, que han seguido un sistema de evaluación continua con exámenes semanales en los cuales se les preguntaba por las horas dedicadas a la preparación de dicho examen. Los resultados obtenidos muestran que la carga real de trabajo autónomo de los estudiantes es bastante menor que la carga que debería derivarse según la definición del crédito ECTS. Se discuten los posibles motivos y se ofrecen alternativas de mejora.

Keywords: ECTS, EEES, carga de trabajo autónomo del estudiante 


\section{Introducción}

El Sistema Europeo de Transferencia y Acumulación de Créditos (ECTS por sus siglas en inglés) constituye uno de los pilares del actual Espacio Europeo de Educación Superior (EEES). El objetivo de este sistema es homogeneizar el concepto de "crédito" en educación superior para que los planes de estudios que siguen este sistema sean comparables y más transparentes. Oficialmente, los créditos ECTS "indican el volumen del aprendizaje a partir de los resultados de aprendizaje y su carga de trabajo asociada" (Comisión Europea 2015).

Esta definición, a la que se acogen las universidades del EEES, favorece la movilidad de los estudiantes así como la planificación y evaluación de las titulaciones, siendo éstos los aspectos más positivos del sistema. Sin embargo, no existe un seguimiento sistematizado del grado en el que esta definición se ajusta a la realidad.

La cuantificación de los créditos ECTS viene por la carga de trabajo asociada que se indica en su definición. Esta "carga de trabajo" es una estimación del tiempo típicamente necesario para que una persona complete todas las actividades planificadas con objeto de alcanzar unos determinados resultados de aprendizaje dentro de un programa formal de educación (Comisión Europea 2015). La carga de trabajo total que oficialmente implica el crédito ECTS varía entre 25 y 30 horas, oscilando entre países, e incluso entre universidades de un mismo país. De estas horas, sólo una parte serían presenciales (habitualmente 10 horas en España). El resto de horas corresponderían a trabajo autónomo por parte del alumno. Sin embargo, algunos estudios previos muestran que, en realidad, la carga de trabajo para el alumno se encuentra generalmente muy por debajo de la derivada de la definición de los créditos ECTS (Jimenez-Munoz 2015; Souto-Iglesias y Baeza-Romero 2018). Analizándola de forma semanal, sólo se alcanzarían los niveles esperados, o incluso se sobrepasarían, solamente en periodos puntuales, habitualmente cercanos a la realización de exámenes (Domenech y col. 2015).

Estas evidencias apuntan a la necesidad de monitorizar de manera sistemática la correspondencia entre la carga de trabajo oficial y la carga de trabajo real de los alumnos, con objeto de mejorar o rediseñar los planes de estudio y los métodos de enseñanza-aprendizaje. De hecho, tras años de aplicación del sistema ECTS se sigue solicitando a las instituciones que ahonden en reforzar el vínculo entre créditos de estudio y carga de trabajo de los estudiantes, y se indica que debería supervisarse si la carga de trabajo de los créditos ECTS en las titulaciones es adecuada y realista (Comisión Europea 2015).

Sin embargo, no tenemos conocimiento de que actualmente se realice un seguimiento regular de la carga de trabajo que implican los créditos ECTS ni por parte de las universidades, ni de organismos supervisores como ANECA en el caso de España, ni tampoco en general por parte de universidades u organismos de otros países pertenecientes al EEES. El único caso específicamente dedicado a esta cuestión que se ha encontrado hasta la fecha es el sistema "ECTS-Monitoring" que están empezando a implementar varias universidades alemanas (University of Düsseldorf 2019; University of Köln 2019; RWTH Aachen University 2019).

Por todo ello, el objetivo de este artículo es comprobar si la carga de trabajo estimada por los créditos ECTS es realista, e implican entre 25 y 30 horas. Para ello, se ha utilizado como caso de estudio una asignatura de primer curso del Grado en 
Administración y Dirección de Empresas que dispone de un sistema de evaluación continua, que es relativamente exigente en cuanto a la dedicación esperada por parte de los alumnos. El artículo se estructura del siguiente modo: el apartado 2 describe el contexto empírico que se ha utilizado para realizar el análisis; el apartado 3 incluye la descripción de la metodología empleada así como de los resultados obtenidos; finalmente, el apartado 4 aporta una discusión en relación a los resultados y resume las conclusiones obtenidas.

\section{Contexto empírico}

La asignatura para la cual se ha realizado el seguimiento de la carga de trabajo autónomo de los estudiantes es Microeconomía I. Se trata de una asignatura obligatoria incluida en el primer cuatrimestre del primer curso del Grado en Administración y Dirección de Empresas de la Universitat Politècnica de València, que consta de un total de 240 ECTS repartidos en cuatro cursos académicos. Según la guía docente, esta asignatura consta de 6 ECTS, que representan 60 horas de docencia presencial y 120 horas de trabajo autónomo. De las 60 horas presenciales, 52 de ellas son en aula y las otras 8 en laboratorio. Generalmente, cada semana lectiva cuenta con 3,5 horas de docencia presencial, que se amplía hasta 5,5 horas en las cuatro semanas en las que hay sesiones de laboratorio programadas.

El sistema de evaluación diseñado para esta asignatura incluye la realización de pruebas semanales acumulativas que suponen un $90 \%$ de la nota final, y de cuatro sesiones de prácticas que suponen el restante $10 \%$.

Las pruebas acumulativas consisten en exámenes escritos que se realizan en el aula durante la mayor parte de semanas lectivas del cuatrimestre. Al finalizar el curso, los alumnos han realizado un total de 12 pruebas, cuya nota constituye el $90 \%$ de la nota final de la asignatura. La última de las pruebas tiene el doble de peso que el resto con el fin de dar a los alumnos la oportunidad de mejorar sus calificaciones. Este sistema de evaluación es obligatorio para todos los alumnos, que no disponen de la opción de realizar un examen final de recuperación.

La duración de las pruebas es de 30 minutos. Cada semana, se alterna entre dos tipos de pruebas distintas: de selección múltiple o de desarrollo. Las pruebas tipo test de selección múltiple incluyen entre 10 y 12 cuestiones con cuatro opciones, de las cuales solamente una es correcta. Las pruebas de desarrollo incluyen entre una y tres cuestiones, que pueden ser tanto problemas prácticos como preguntas teóricas. Estas pruebas son de naturaleza acumulativa, esto es, cubren todo el contenido visto en clase hasta el momento de realizar el examen. El objetivo de plantear estos exámenes como acumulativos es contribuir a que los alumnos consoliden el conocimiento adquirido sobre la asignatura, dado que tienen que repasar todos los contenidos regularmente.

La alta frecuencia en la realización de pruebas viene acompañada también de una alta frecuencia en la publicación de las soluciones y de las notas, para que los alumnos reciban una rápida retroalimentación que les permita trabajar en sus puntos débiles de cara a la siguiente prueba.

Con este sistema de evaluación, se fuerza a los alumnos para que trabajen en la asignatura de manera regular, estando por tanto alineado con la definición de ECTS que se emplea en el sistema de educación superior español según la cual el trabajo 
autónomo por parte del alumno debería suponer la mayor carga (y una carga muy superior a las horas lectivas). En la siguiente sección, se analiza si la carga de trabajo planteada por los ECTS se corresponde con la carga que realmente suponen para los estudiantes que, en este caso, han seguido un sistema de evaluación frecuente y, así, relativamente exigente.

\section{Metodología y resultados}

Para obtener los datos con los que realizar el estudio, en cada prueba se preguntó a los alumnos cuánto tiempo habían dedicado para prepararla. Desde el primer momento, los alumnos fueron informados de que el objetivo era realizar un estudio de la carga de trabajo que supone para ellos y que su participación es opcional. Se les comunicó también que no tendría ninguna repercusión en su nota, y que el análisis de sus respuestas se realizaría una vez finalizado el curso.

De los 50 alumnos matriculados en el grupo de Alto Rendimiento Académico de Microeconomía I, en el que se ha desarrollado este estudio, 29 de ellos contestaron a la pregunta sobre el tiempo dedicado en 9 o más pruebas. Éstas son las respuestas que se analizarán a continuación. El resto de respuestas, pertenecientes a alumnos que respondieron pocas veces (o ninguna), se han eliminado del análisis.

La figura 1 muestra la evolución de las horas dedicadas a cada una de las 12 pruebas semanales realizadas. Como es de esperar, el tiempo dedicado a cada prueba va aumentando a medida que aumenta la cantidad de materia impartida. La media de tiempo dedicado aumenta desde los 102 minutos de la primera prueba, hasta los 367 de la última. La dispersión del tiempo dedicado también aumenta a medida que lo hace el tiempo empleado. Recordemos que las 3,5 horas de clase semanales, esto es, 210 minutos por semana, deberían corresponder a una carga de trabajo autónoma de entre 315 y 420 minutos (entre 5,25 y 7 horas). Sólo dos de las pruebas alcanzan medias de dedicación en este intervalo, correspondientes a las pruebas 10 y 12 .

Si analizamos la mediana, en lugar de la media, como indicador de la carga de trabajo típica, los resultados se alejan todavía más de la definición del crédito ECTS. La mediana de carga de trabajo varía entre los 90 minutos de la primera prueba y los 240 minutos de la última. Así, ninguna de las pruebas representó una carga de trabajo acorde a la definición.

A continuación, se analizan las horas totales dedicadas al estudio de la asignatura, sumando las horas declaradas por cada estudiante. La figura 2 muestra el histograma de los estudiantes según el tiempo total de estudio. Éste presenta una distribución asimétrica hacia la derecha, con relativamente pocos estudiantes que le dedican un tiempo superior a la media. El intervalo con mayor número de estudiantes es el que incluye aquéllos que le han dedicado entre 24 y 30 horas de estudio total. Recordemos que, dado que las sesiones de aula constituyen 52 horas, cabría esperar que los alumnos dedicaran entre 78 y 104 horas (es decir, entre 1,5 y 2 veces más que las horas presenciales siguiendo la definición del crédito ECTS). Según los datos de la figura 2, sólo dos de los 28 estudiantes que contestaron se encuentran en esta franja. Un estudiante está por encima, mientras que los otros 25 están por debajo. No sólo eso, sino que la moda se encuentra en menos de la mitad del tiempo típico previsto. 


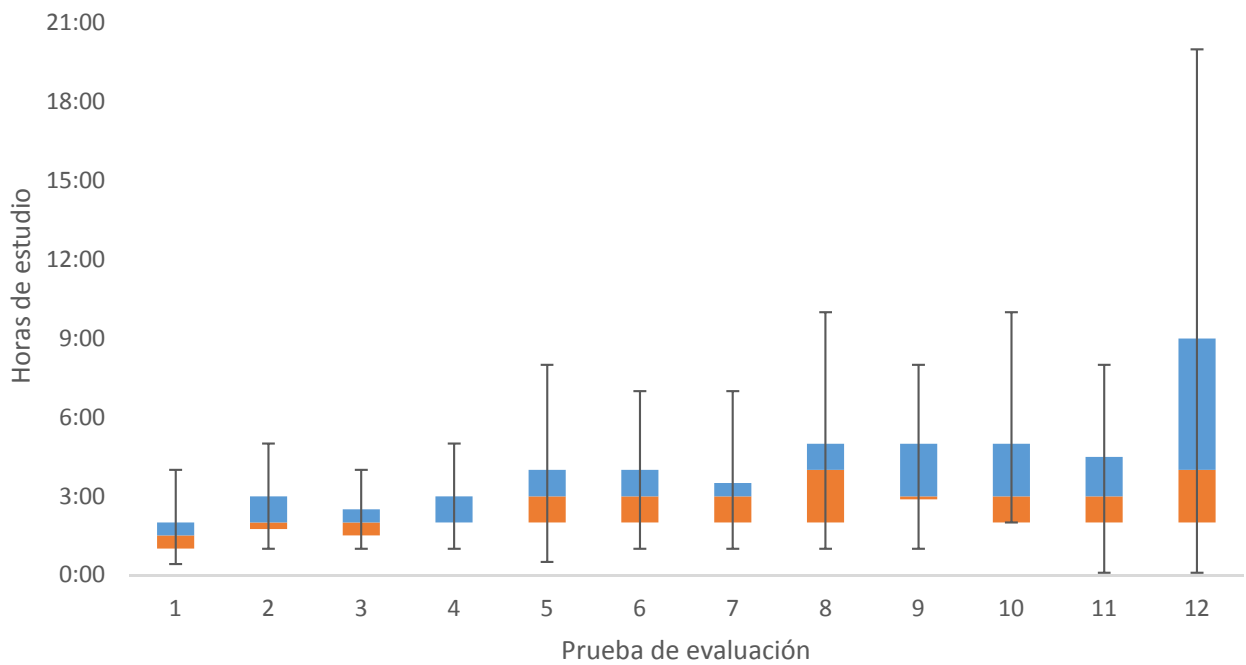

Fig. 1: Distribución de las horas dedicadas a cada prueba semanal. Nota: no se muestran los valores extremos (fuera de 1,5 veces el rango intercuartil).

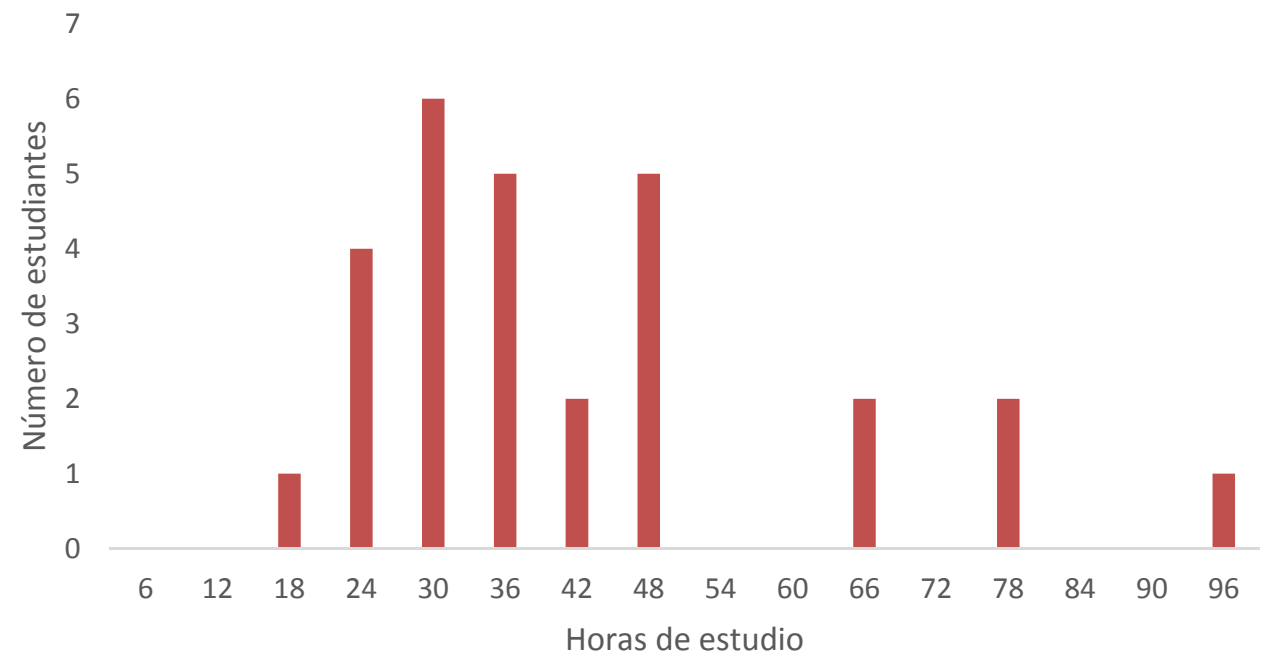

Fig. 2: Histograma de las horas totales dedicadas por los alumnos al estudio de la asignatura. Nota: Se ha omitido la representación de un estudiante que declaró haber dedicado 180 horas. 


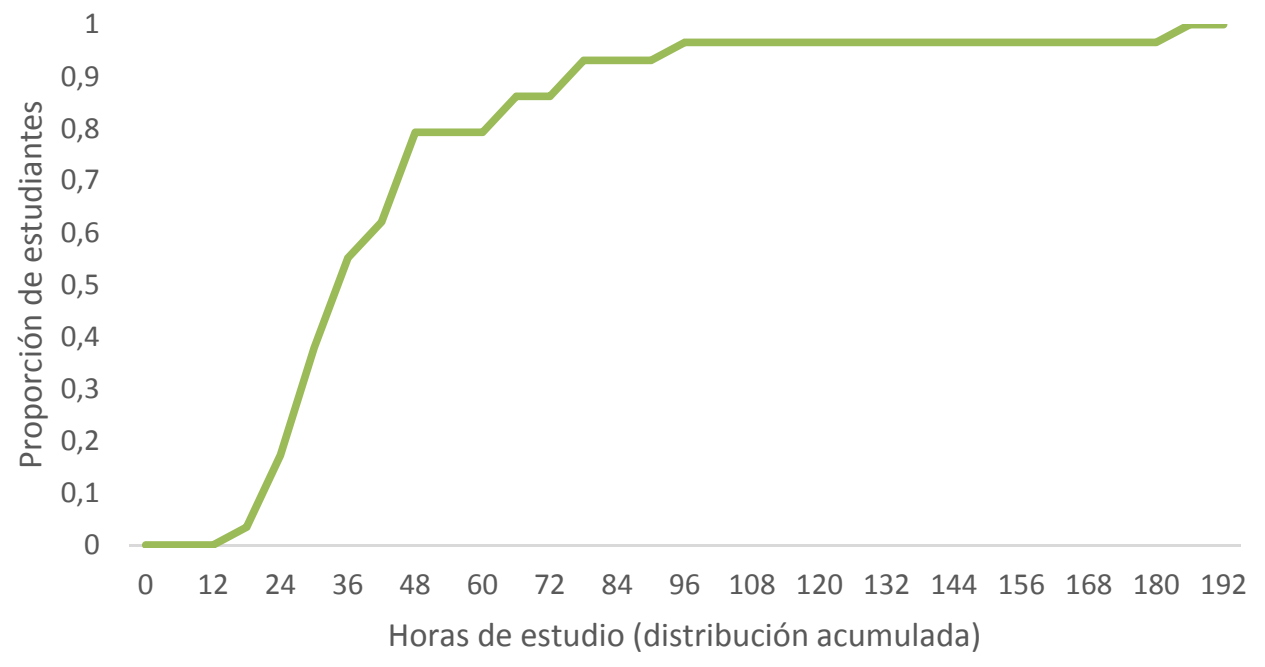

Fig. 3: Distribución acumulada del número de horas dedicadas al estudio de la asignatura.

Finalmente, la figura 3 ilustra la distribución acumulada del tiempo dedicado al estudio de Microeconomía I. Se puede observar que la mitad de estudiantes dedican menos de 36 horas de trabajo autónomo, es decir, que le dedican menos de un $46 \%$ del tiempo mínimo que establece la definición de crédito ECTS (78 horas). En esta figura también se puede ver que el $86 \%$ de los alumnos le dedica menos de este tiempo.

\section{Discusión y conclusiones}

Los resultados aquí mostrados destacan que la carga de trabajo que esta asignatura representa se encuentra alejada de la declarada en la guía docente y de la que se derivaría de la definición del crédito ECTS.

Existen varias explicaciones posibles para la desviación manifiesta. Por una parte, podría pensarse que se trata de un caso puntual, que afecta sólo a un grupo, a una asignatura o a una titulación. Las impresiones que los docentes recibimos de los alumnos hacen referencia al hecho de que es una asignatura exigente, a la que le deben dedicar importantes esfuerzos durante todo el curso. Asimismo, la divergencia observada es consistente, y cercana en valores absolutos, con las descritas por otros autores en estudios de grado en España (véase, por ejemplo, Domenech y col. 2015; Souto-Iglesias y Baeza-Romero 2018). Aunque los resultados no son generalizables, pensamos que podría deberse a un defecto estructural de la implantación de los créditos ECTS (al menos en España), en lugar de una deficiencia puntual de un grupo, asignatura, o titulación.

Por otra parte, podría haber alguna deficiencia en la recogida de la información, de forma que los datos recabados no reflejen fielmente el tiempo dedicado al trabajo autónomo. Sin embargo, la frecuencia cuasi semanal de las pruebas deja pocas semanas sin recogida de información, a la vez que no parece existir incentivo para declarar menos horas de las dedicadas, sino más bien al contrario. 
Se vislumbran varias alternativas para alinear la definición de créditos ECTS con la carga de trabajo típica del estudiante:

Revisar la relación entre crédito ECTS y horas de clase. Una opción sería aumentar las horas presenciales incluidas en cada crédito ECTS. Con los datos encontrados en este y otros estudios, sería necesario impartir más de 20 horas de clase por cada crédito para que representen la carga de trabajo objetivo. Seguramente se trate de una carga presencial excesiva tanto para alumnos como para docentes, si la dedicación docente se sigue midiendo en créditos ECTS y no en horas. De forma comparada con otros países del EEES, España se encuentra entre los que más horas de clase presencial asigna a cada ECTS, por lo que no parece que ésta sea la solución más conveniente.

Revisar la definición de crédito ECTS. Si la definición actual no es realista, es decir, si la relación entre créditos ECTS y horas de trabajo del estudiante no se cumplen en una amplia variedad de contextos (asignaturas, titulaciones, y países), sería conveniente reconsiderar la definición de crédito ECTS para adecuarla a la realidad.

Cambiar la relación entre horas presenciales y horas de trabajo autónomo. Un motivo por el que los créditos ECTS no reflejan la carga del estudiante puede ser por el hecho de que los docentes no exijamos el suficiente trabajo autónomo a los alumnos. Un aumento del uso de metodologías activas de aprendizaje podría fomentar la necesidad del estudiante de dedicarle más tiempo por su cuenta por cada hora de clase recibida. Esto implicaría cambiar de forma sustancial y generalizada la forma en la que llevamos las clases.

En cualquier caso, se pone de manifiesto la necesidad de hacer un seguimiento cercano de la carga de trabajo que cada crédito ECTS representa para el alumnado. La definición del crédito ECTS tiene implicaciones en varios aspectos de la organización académica:

- El crédito ECTS, como unidad de transferencia, debe ser consistente entre instituciones. En caso contrario, se estarían utilizando unidades heterogéneas en la convalidación y homologación de estudios entre distintas instituciones del EEES. Y la mejor forma de lograrlo es que mida fielmente aquello para lo que se definió.

- Hace falta evaluar los créditos y ajustar las titulaciones de forma acorde. Si dos materias con la misma carga horaria presencial resultan en distinta carga de trabajo para los alumnos, la materia con más carga debería ser reconocida con más créditos. Asimismo, se deberían reducir los créditos de aquellas materias que no requieran de tanto trabajo.

- Si de forma persistente estamos exigiendo menos trabajo a los alumnos de lo que deberíamos, estamos devaluando nuestros títulos. La falta de carga de trabajo podría ser compensada con nuevas materias o con otras actividades obligatorias que complementen a las materias actuales.

Por último, reconocer las limitaciones del trabajo realizado. Se trata de un estudio de pequeña escala por lo que los resultados se deben tomar con mucha precaución. En cualquier caso, se quiere hacer notar la necesidad de la evaluación de la carga de tra- 
bajo para conocer realmente qué significa un crédito ECTS en nuestras titulaciones. Es fundamental hacer el seguimiento de horas de trabajo del estudiante para autoevaluarnos como gestores del aprendizaje de los alumnos y corregir nuestra actividad docente.

\section{Referencias bibliográficas}

Comisión Europea (2015). Guía de Uso del ECTS. Luxemburgo: Oficina de Publicaciones de la Unión Europea, 2015. ISBN: 978-92-79-58516-6.

Domenech, Gisbert y col. (2015). "Seguimiento del trabajo no presencial: retroalimentación y optimización". En: In-Red 2015-CONGRESO NACIONAL DE INNOVACIÓN EDUCATIVA Y DE DOCENCIA EN RED. Editorial Universitat Politècnica de València.

Jimenez-Munoz, Antonio (2015). "Flipping lectures: analysing student workload in EMI contexts". En: Procedia-Social and Behavioral Sciences 212, págs. 35-41.

RWTH Aachen University (2019). ECTS-Monitoring. https://www.hrk-nexus.de/ fileadmin/redaktion/hrk-nexus/07-Downloads/07-01-Tagungen/07-0158-Monitoring-III/Sander_HauffeWaschbuesch.pdfl, consultado el 22 de marzo de 2019.

Souto-Iglesias, Antonio y María Teresa Baeza-Romero (2018). "A probabilistic approach to student workload: empirical distributions and ECTS". En: Higher Education 76.6, págs. 1007-1025.

University of Düsseldorf (2019). ECTS Monitoring. https : / / hs-duesseldorf . de/hochschule/verwaltung/strategie_innovation/QMEva/Seiten/ects monitoring. aspx, consultado el 22 de marzo de 2019.

University of Köln (2019). Landesweites ECTS-Monitoring. https : //www · portal . uni-koeln.de/13648.html, consultado el 22 de marzo de 2019. 